\title{
On the Solution Existence of Variational-Like Inequalities Problems for Weakly Relaxed $\eta-\alpha$ Monotone Mapping
}

\author{
Marwan Amin Kutbi ${ }^{1}$ and Wutiphol Sintunavarat ${ }^{2}$ \\ ${ }^{1}$ Department of Mathematics, King Abdulaziz University, P.O. Box 80203, Jeddah 21589, Saudi Arabia \\ ${ }^{2}$ Department of Mathematics and Statistics, Faculty of Science and Technology, Thammasat University Rangsit Center, \\ Pathum Thani 12121, Thailand
}

Correspondence should be addressed to Wutiphol Sintunavarat; wutiphol@mathstat.sci.tu.ac.th

Received 10 September 2013; Accepted 20 October 2013

Academic Editor: S. Romaguera

Copyright (C) 2013 M. A. Kutbi and W. Sintunavarat. This is an open access article distributed under the Creative Commons Attribution License, which permits unrestricted use, distribution, and reproduction in any medium, provided the original work is properly cited.

\begin{abstract}
We introduce two new concepts of weakly relaxed $\eta-\alpha$ monotone mappings and weakly relaxed $\eta-\alpha$ semimonotone mappings. Using the KKM technique, the existence of solutions for variational-like problems with weakly relaxed $\eta-\alpha$ monotone mapping in reflexive Banach spaces is established. Also, we obtain the existence of solution for variational-like problems with weakly relaxed $\eta-\alpha$ semimonotone mappings in arbitrary Banach spaces by using the Kakutani-Fan-Glicksberg fixed-point theorem.
\end{abstract}

\section{Introduction}

The variational inequality theory provides us with a simple, natural, unified, and elegant framework to study a wide class of linear and nonlinear problems arising in many fields, such as mechanics, engineering sciences, elasticity, optimization, control, programming, economics, transportation, oceanography, and regional. Because of their wide applicability, various extensions and generalizations of the classical variational inequality problem have been proposed and studied in recent years. Variational-like inequalities problems is one of cornerstone in this field. Some special case of generalized variational inequalities and variational-like inequalities have been studied by several authors including Bai et al. [1], Chang et al. [2], dos Santos et al. [3], Xiao and Huang [4], Zhao and Xia $[5,6]$, and references therein.

It is well known that the monotonicity and generalized monotonicity play an important role of the study in variational inequality theory. In recent years, a substantial number of papers on existence results for solving variational inequality problems and variational-like inequality problems based on different generalization of monotonicity such as pseudomonotonicity, quasimonotonicity, relaxed monotonicity, semimonotonicity, and $p$-monotonicity (see [7-15]) appeared. In [16], Fang and Huang introduced a new concept of relaxed $\eta-\alpha$ monotonicity and obtained the existence of solutions for variational-like inequalities with relaxed $\eta-\alpha$ monotone mappings in reflexive Banach spaces. Recently, Sintunavarat [17] established the existence of solution of mixed equilibrium problem with the weakly relaxed $\alpha$-monotone bi-function in Banach spaces.

Inspired and motivated by the work of Fang and Huang [16] and Sintunavarat [17], in this paper we introduce the two new concepts of weakly relaxed $\eta-\alpha$ monotone mappings and weakly relaxed $\eta-\alpha$ semimonotone mappings as well as two classes of variational-like inequalities with weakly relaxed $\eta-\alpha$ monotone mappings and weakly relaxed $\eta-\alpha$ semimonotone mappings. By using the KKM technique, we study some existence of solutions for variational-like inequalities with weakly relaxed $\eta-\alpha$ monotone mappings in reflexive Banach spaces. We also obtain the solvability of variational-like inequalities with weakly relaxed $\eta-\alpha$ semimonotone mappings in arbitrary Banach spaces by using the Kakutani-FanGlicksberg fixed-point theorem. Our results in this paper 
extend and improve the results of Fang and Huang [16] and many results in the literature.

\section{Preliminaries}

In this paper, unless otherwise specified, $K$ is a nonempty closed convex subset of a real reflexive Banach space $E$ with dual space $E^{*}$. The following basic knowledge will be useful in our work.

Definition 1 (see [18]). Let $T: K \rightarrow E^{*}$ and $\eta: K \times K \rightarrow E$ be two mappings. A mapping $T$ is said to be $\eta$-hemicontinuous if, for any fixed $x, y \in K$, the mapping $f:[0,1] \rightarrow \mathbb{R}$ defined by

$$
f(t)=\langle T(t y+(1-t) x), \eta(y, x)\rangle
$$

is continuous at $0^{+}$.

Definition 2 (see [16]). Let $T: K \rightarrow E^{*}$ and $\eta: K \times K \rightarrow E$ be two mappings. A mapping $T$ is said to be $\eta$-coercive with respect to a proper function $f: K \rightarrow \mathbb{R} \cup\{\infty\}$ if there exists $x_{0} \in K$ such that

$$
\frac{\left[\left\langle T x-T x_{0}, \eta\left(x, x_{0}\right)\right\rangle+f(x)-f\left(x_{0}\right)\right]}{\left\|\eta\left(x, x_{0}\right)\right\|} \rightarrow \infty,
$$

whenever $\|x\|$ is large enough.

Remark 3. If $f=\delta_{K}$, where $\delta_{K}$ is the indicator function of $K$, then Definition 2 coincides with the definition of $\eta$-coercivity in the sense of Yang and Chen [15].

Definition 4 (see [16]). A mapping $T: K \rightarrow E^{*}$ is said to be relaxed $\eta$ - $\alpha$ monotone if there exist a function $\eta: K \times K \rightarrow E$ and $\alpha: E \rightarrow \mathbb{R}$ with $\alpha(t z)=t^{p} \alpha(z)$ for all $t>0$ and $z \in E$ such that

$$
\langle T x-T y, \eta(x, y)\rangle \geq \alpha(x-y), \quad \forall x, y \in K,
$$

where $p>1$ is a constant.

Remark 5. (1) If $\eta(x, y)=x-y$ for all $x, y \in K$, then (3) reduces to

$$
\langle T x-T y, x-y\rangle \geq \alpha(x-y), \quad \forall x, y \in K,
$$

and $T$ is said to be $\alpha$-monotone.

(2) If $\eta(x, y)=x-y$ for all $x, y \in K$ and $\alpha(z)=k\|z\|^{p}$, where $k>0$ and $p>1$, then (3) becomes

$$
\langle T x-T y, x-y\rangle \geq k\|x-y\|^{p}, \quad \forall x, y \in K,
$$

and $T$ is said to be $p$-monotone (see in $[14,19,20]$ ).

(3) Every monotone mapping is relaxed $\eta-\alpha$ monotone with $\eta(x, y)=x-y$ for all $x, y \in K$ and $\alpha \equiv 0$.

Definition 6. Let $F: K \rightarrow 2^{E}$ be a set-valued mapping. Then, $F$ is said to be KKM mapping if, for any finite subset $\left\{y_{1}, y_{2}, \ldots, y_{n}\right\}$ of $K$, we have $\operatorname{co}\left\{y_{1}, y_{2}, \ldots, y_{n}\right\} \subset$ $\bigcup_{i=1}^{n} F\left(y_{i}\right)$, where $\operatorname{co}\left\{y_{1}, y_{2}, \ldots, y_{n}\right\}$ denotes the convex hull of $\left\{y_{1}, y_{2}, \ldots, y_{n}\right\}$.
Remark 7. Let $F, G: K \rightarrow 2^{E}$. If $F$ is KKM mapping and $F(y) \subset G(y)$ for all $y \in K$, then $G$ is also KKM mapping.

Lemma 8 (see [21]). Let $M$ be a nonempty subset of a Hausdorff topological vector space $X$, and let $F: M \rightarrow 2^{X}$ be a KKM mapping. If $F(y)$ is closed in $X$ for all $y \in M$ and compact for some $y \in M$, then $\bigcap_{y \in M} F(y) \neq \emptyset$.

\section{Variational-Like Inequalities Problems with Weakly Relaxed $\eta-\alpha$ Monotone Mapping}

In this section, we introduce the new class of mapping which generalizes several classes. Using KKM technique, we study and prove the existence of solutions for variational-like inequalities problems with mapping in such class in Banach spaces.

Definition 9. A mapping $T: K \rightarrow E^{*}$ is said to be weakly relaxed $\eta$ - $\alpha$ monotone if there exist a function $\eta: K \times K \rightarrow E$ and $\alpha: E \rightarrow \mathbb{R}$ with

$$
\begin{gathered}
\lim _{t \rightarrow 0^{+}} \alpha(t x)=0, \\
\lim _{t \rightarrow 0^{+}} \frac{d}{d t} \alpha(t x)=0,
\end{gathered}
$$

for all $t>0$ and $x \in E$ such that

$$
\langle T x-T y, \eta(x, y)\rangle \geq \alpha(x-y), \quad \forall x, y \in K .
$$

Remark 10 . We obtain that the relaxed $\eta-\alpha$ monotonicity implies weakly relaxed $\eta-\alpha$ monotonicity. So, class of relaxed $\eta-\alpha$ monotone mapping is subclass of weakly relaxed $\eta-\alpha$ monotone mapping class. Also, we get that classes of relaxed $\alpha$ monotone mapping, $p$-monotone mapping, and monotone mapping are subclass of weakly relaxed $\eta-\alpha$ monotone mapping class.

Theorem 11. Let $T: K \rightarrow E^{*}$ be an $\eta$-hemicontinuous and weakly relaxed $\eta-\alpha$ monotone and let $f: K \rightarrow \mathbb{R} \cup\{\infty\}$ be a proper convex function. Suppose that the following conditions hold:

(a) $\eta(x, x)=0$ for all $x \in K$;

(b) for any fixed $y, z \in K$, the mapping $x \mapsto\langle T z, \eta(x, y)\rangle$ is convex.

Then, the following problems (9) and (10) are equivalent:

$$
\begin{gathered}
\text { find } x \in K \\
\text { such that }\langle T x, \eta(y, x)\rangle+f(y)-f(x) \geq 0, \\
\forall y \in K,
\end{gathered}
$$

find $x \in K$

such that $\langle T y, \eta(y, x)\rangle+f(y)-f(x) \geq \alpha(y-x)$,

$\forall y \in K$. 
Proof. Suppose that (9) has a solution. So, there exists $x \in K$ such that

$$
\langle T x, \eta(y, x)\rangle+f(y)-f(x) \geq 0, \quad \forall y \in K .
$$

Since $T$ is weakly relaxed $\eta$ - $\alpha$ monotone, we have

$$
\langle T y-T x, \eta(y, x)\rangle \geq \alpha(y-x), \quad \forall y \in K,
$$

and then

$$
\begin{aligned}
\langle T y, & \eta(y, x)\rangle+f(y)-f(x) \\
& \geq\langle T x, \eta(y, x)\rangle+\alpha(y-x)+f(y)-f(x) \\
& \geq \alpha(y-x), \quad \forall y \in K .
\end{aligned}
$$

Therefore, $x \in K$ is a solution of problem (10).

Conversely, suppose that $x \in K$ is a solution of problem (10) and $y$ is any point in $K$ with $f(y)<\infty$. For $t \in(0,1)$, we let $y_{t}:=(1-t) x+t y$. From $(10)$, we get that $f(x)<\infty$. It follows from $K$ being convex that $y_{t} \in K$. From (10), we have

$$
\begin{aligned}
& \left\langle T y_{t}, \eta\left(y_{t}, x\right)\right\rangle+f\left(y_{t}\right)-f(x) \geq \alpha\left(y_{t}-x\right) \\
& =\alpha(t(y-x)),
\end{aligned}
$$

and thus

$$
f\left(y_{t}\right)-f(x) \geq \alpha(t(y-x))-\left\langle T y_{t}, \eta\left(y_{t}, x\right)\right\rangle .
$$

The convexity of $f$ implies that

$$
\begin{aligned}
f\left(y_{t}\right)-f(x) & =f((1-t) x+t y)-f(x) \\
& \leq t(f(y)-f(x)) .
\end{aligned}
$$

From (15) and (16), we get

$$
t(f(y)-f(x)) \geq \alpha(t(y-x))-\left\langle T y_{t}, \eta\left(y_{t}, x\right)\right\rangle .
$$

By the assumption (b), we have

$$
\begin{aligned}
\left\langle T y_{t}, \eta\left(y_{t}, x\right)\right\rangle & =\left\langle T y_{t}, \eta((1-t) x+t y, x)\right\rangle \\
& \leq(1-t)\left\langle T y_{t}, \eta(x, x)\right\rangle+t\left\langle T y_{t}, \eta(x, y)\right\rangle \\
& =t\left\langle T y_{t}, \eta(y, x)\right\rangle \\
& =t\langle T((1-t) x+t y), \eta(y, x)\rangle .
\end{aligned}
$$

It follows from (17) and (18) that

$$
\begin{aligned}
& t\langle T((1-t) x+t y), \eta(y, x)\rangle+t(f(y)-f(x)) \\
& \quad \geq \alpha(t(y-x))
\end{aligned}
$$

that is

$$
\begin{aligned}
& \langle T((1-t) x+t y), \eta(y, x)\rangle+f(y)-f(x) \\
& \quad \geq \frac{\alpha(t(y-x))}{t},
\end{aligned}
$$

for all $y \in K$. Taking $t \rightarrow 0^{+}$in the previous inequality and using $\eta$-hemicontinuity of $T$, we get

$$
\langle T x, \eta(y, x)\rangle+f(y)-f(x) \geq \lim _{t \rightarrow 0^{+}} \frac{\alpha(t(y-x))}{t} .
$$

From (6), we get $\lim _{t \rightarrow 0^{+}}(\alpha(t(y-x)) / t)$ is indeterminate form. Using L' Hôpital's rule, we obtain that

$$
\langle T x, \eta(y, x)\rangle+f(y)-f(x) \geq \lim _{t \rightarrow 0^{+}} \frac{(d / d t) \alpha(t(y-x))}{1} .
$$

By property (7) of weakly relaxed $\eta-\alpha$ monotone of $T$, we have

$$
\langle T x, \eta(y, x)\rangle+f(y)-f(x) \geq 0,
$$

for all $y \in K$ with $f(y)<\infty$. In case of $f(y)=\infty$, the relation

$$
\langle T x, \eta(y, x)\rangle+f(y)-f(x) \geq 0
$$

is trivial. Therefore, $x \in K$ is also a solution of problem (9).

Theorem 12. Let $K$ be a nonempty bounded closed convex subset of a real reflexive Banach space $E$, and let $E^{*}$ be the dual space of E. Suppose that $T: K \rightarrow E^{*}$ is an $\eta$-hemicontinuous and weakly relaxed $\eta-\alpha$ monotone mapping and $f: K \rightarrow$ $\mathbb{R} \cup\{\infty\}$ is a proper convex lower semicontinuous function. If the following conditions hold:

(a) $\eta(x, y)+\eta(y, x)=0$ for all $x, y \in K$,

(b) for any fixed $y, z \in K$, the mapping $x \mapsto\langle T z, \eta(x, y)\rangle$ is convex and lower semicontinuous function,

(c) $\alpha$ is weakly lower semicontinuous; that is, for any net $\left\{x_{\beta}\right\}$, we have that $x_{\beta}$ converges to $x$ in $\sigma\left(E, E^{*}\right)$ implies that $\alpha(x) \leq \lim \inf \alpha\left(x_{\beta}\right)$,

then the problem (9) is solvable.

Proof. Consider the set valued mapping $F: K \rightarrow 2^{E}$ defined by

$$
F(y)=\{x \in K:\langle T x, \eta(y, x)\rangle+f(y)-f(x) \geq 0\},
$$

for all $y \in K$.

It is easy to see that $x \in K$ solves the problem (9); that is,

$$
\langle T x, \eta(y, x)\rangle+f(y)-f(x) \geq 0, \quad \forall y \in K,
$$

if and only if $x \in \bigcap_{y \in K} F(y)$. Thus, it is sufficient to prove that $\bigcap_{y \in K} F(y) \neq \emptyset$.

Next, we show that $F$ is a KKM mapping. Assume the contrary, then there exists $\left\{y_{1}, y_{2}, \ldots, y_{m}\right\} \subset K$ such that $\operatorname{co}\left\{y_{1}, y_{2}, \ldots, y_{m}\right\} \not \subset \bigcup_{i=1}^{m} F\left(y_{i}\right)$. This implies that there exists $y_{0} \in \operatorname{co}\left\{y_{1}, y_{2}, \ldots, y_{m}\right\}$ such that $y_{0}=\sum_{i=1}^{m} t_{i} y_{i}$, where $t_{i} \geq 0$, $i=1,2, \ldots, m$ and $\sum_{i=1}^{m} t_{i}=1$, but $y_{0} \notin \bigcup_{i=1}^{m} F\left(y_{i}\right)$.

From (25), we have

$$
\left\langle T y_{0}, \eta\left(y_{i}, y_{0}\right)\right\rangle+f\left(y_{i}\right)-f\left(y_{0}\right)<0, \quad \text { for } i=1,2, \ldots, m \text {. }
$$


By (b) and (25), we obtain that

$$
\begin{aligned}
0 & =\left\langle T y_{0}, \eta\left(y_{0}, y_{0}\right)\right\rangle \\
& =\left\langle T y_{0}, \eta\left(\sum_{i=1}^{m} t_{i} y_{i}, y_{0}\right)\right\rangle \\
& \leq \sum_{i=1}^{m} t_{i}\left\langle T y_{0}, \eta\left(y_{i}, y_{0}\right)\right\rangle \\
& <\sum_{i=1}^{m} t_{i}\left[f\left(y_{0}\right)-f\left(y_{i}\right)\right] \\
& =f\left(y_{0}\right)-\sum_{i=1}^{m} t_{i} f\left(y_{i}\right) \\
& \leq f\left(y_{0}\right)-f\left(y_{0}\right) \\
& =0,
\end{aligned}
$$

which is a contradiction. Therefore, $F$ is a KKM mapping. by

Now, we define another set-valued mapping $G: K \rightarrow 2^{E}$

$$
G(y)=\{x \in K:\langle T x, \eta(y, x)\rangle+f(y)-f(x) \geq \alpha(y-x)\}
$$

for all $y \in K$.

Next, we will claim that $F(y) \subset G(y)$ for all $y \in K$. For each $y \in K$, let $x \in F(y)$; then,

$$
\langle T x, \eta(y, x)\rangle+f(y)-f(x) \geq 0 .
$$

From the weakly relaxed $\eta-\alpha$ monotonicity of $T$, we get

$$
\begin{aligned}
& \langle T y, \eta(y, x)\rangle+f(y)-f(x) \\
& \quad \geq \alpha(y-x)+[\langle T x, \eta(y, x)\rangle+f(y)-f(x)] \\
& \quad \geq \alpha(y-x) .
\end{aligned}
$$

This implies that $x \in G(y)$ and hence $F(y) \subset G(y)$ for all $y \in K$. So, $G$ is also a KKM mapping.

By assumption, $x \mapsto\langle T y, \eta(x, y)\rangle$ and $f$ are two convex lower-semicontinuous functions. Then it is easy to see that they are both weakly lower semicontinuous. From the definition of $G$ and the weakly lower semicontinuity of $\alpha$, we get that $G(y)$ is weakly closed for all $y \in K$. Since $K$ is closed bounded and convex, it also is weakly compact, and then $G(y)$ is weakly compact in $K$ for each $y \in K$. From Lemma 8 and Theorem 11, we obtain that

$$
\bigcap_{y \in K} F(y)=\bigcap_{y \in K} G(y) \neq \emptyset .
$$

So, there exists $x \in K$, such that

$$
\langle T x, \eta(y, x)\rangle+f(y)-f(x) \geq 0, \quad \forall y \in K ;
$$

that is, problem (9) has a solution. This completes the proof.
We know that the relaxed $\eta$ - $\alpha$ monotonicity implies the weakly relaxed $\eta-\alpha$ monotonicity. Therefore, Theorem 12 can be deduced to the following corollary.

Corollary 13. Let $K$ be a nonempty bounded closed convex subset of a real reflexive Banach space $E$, and let $E^{*}$ be the dual space of $E$. Suppose that $T: K \rightarrow E^{*}$ is an $\eta$-hemicontinuous and relaxed $\eta-\alpha$ monotone mapping and $f: K \rightarrow \mathbb{R} \cup\{\infty\}$ is a proper convex lower semicontinuous function. If the following conditions hold:

(a) $\eta(x, y)+\eta(y, x)=0$ for all $x, y \in K$,

(b) for any fixed $y, z \in K$, the mapping $x \mapsto\langle T z, \eta(x, y)\rangle$ is convex and lower semicontinuous function,

(c) $\alpha$ is weakly lower semicontinuous,

then the problem (9) is solvable.

Remark 14. Since the monotonicity, $p$-monotonicity, and relaxed $\alpha$-monotonicity imply relaxed $\eta$ - $\alpha$ monotonicity, we can be applying Corollary 13 to the other problems for the mapping satisfies these property.

Next, we study and prove that result for the case of $K$ is unbounded set.

Theorem 15. Let $K$ be a nonempty unbounded closed convex subset of a real reflexive Banach space $E$, and let $E^{*}$ be the dual space of $E$. Suppose that $T: K \rightarrow E^{*}$ is an $\eta$-hemicontinuous and weakly relaxed $\eta-\alpha$ monotone mapping and $f: K \rightarrow \mathbb{R} \cup$ $\{\infty\}$ is a proper convex lower semicontinuous function. If the following conditions hold:

(a) $\eta(x, y)+\eta(y, x)=0$ for all $x, y \in K$,

(b) for any fixed $y, z \in K$, the mapping $x \mapsto\langle T z, \eta(x, y)\rangle$ is convex and lower semicontinuous function,

(c) $\alpha$ is weakly lower semicontinuous,

(d) $T$ is weakly $\eta$-coercive with respect to $f$; that is, there exists $x_{0} \in K$ such that

$$
\left\langle T x, \eta\left(x_{0}, x\right)\right\rangle+f\left(x_{0}\right)-f(x)<0,
$$

whenever $x \in K$ and $\|x\|$ large enough,

then the problem (9) is solvable.

Proof. For $\epsilon>0$, define $K_{\epsilon}:=\{y \in K:\|y\| \leq \epsilon\}$. Consider the following problem:

$$
\begin{aligned}
& \text { find } x \in K_{\epsilon} \\
& \text { such that }\left\langle T x_{\epsilon}, \eta\left(y, x_{\epsilon}\right)\right\rangle+f(y)-f\left(x_{\epsilon}\right) \geq 0, \\
& \forall y \in K_{\epsilon} .
\end{aligned}
$$

Since $K_{\epsilon}$ is bounded, by Theorem 12, we get that the problem (35) has at least one solution $x_{\epsilon} \in K_{\epsilon}$.

For $x_{0}$ in the weakly $\eta$-coercivity condition (d), we fixed $\epsilon>\left\|x_{0}\right\|$. From (35), we can find that $x_{\epsilon} \in K_{\epsilon}$ such that

$$
\left\langle T x_{\epsilon}, \eta\left(x_{0}, x_{\epsilon}\right)\right\rangle+f\left(x_{0}\right)-f\left(x_{\epsilon}\right) \geq 0 .
$$


Since $x_{\epsilon} \in K_{\epsilon}$, we have $\left\|x_{\epsilon}\right\| \leq \epsilon$. If $\left\|x_{\epsilon}\right\|=\epsilon$ for all $\epsilon$, we may choose $\epsilon$ large enough so that the weakly $\eta$-coercivity condition (d) implies that

$$
\left\langle T x_{\epsilon}, \eta\left(x_{0}, x_{\epsilon}\right)\right\rangle+f\left(x_{0}\right)-f\left(x_{\epsilon}\right)<0,
$$

which contradicts (36). Therefore, there exists $\epsilon$ such that $\left\|x_{\epsilon}\right\|<\epsilon$.

For each $y \in K$, we can choose $0<t<1$ such that $t y+$ $(1-t) x_{\epsilon} \in K_{\epsilon}$. From (35) and the fact that $t y+(1-t) x_{\epsilon} \in K_{\epsilon}$, we have

$$
\begin{aligned}
& \left\langle T x_{\epsilon}, \eta\left(t y+(1-t) x_{\epsilon}, x_{\epsilon}\right)\right\rangle+f\left(t y+(1-t) x_{\epsilon}\right)-f\left(x_{\epsilon}\right) \\
& \quad \geq 0 .
\end{aligned}
$$

By the above inequality and the convexity of $f$ and mapping in (b), we get

$$
\begin{aligned}
& t\left\langle T x_{\epsilon}, \eta\left(y, x_{\epsilon}\right)\right\rangle+t f(y)-t f\left(x_{\epsilon}\right) \\
&= t\left\langle T x_{\epsilon}, \eta\left(y, x_{\epsilon}\right)\right\rangle+(1-t)\left\langle T x_{\epsilon}, \eta\left(x_{\epsilon}, x_{\epsilon}\right)\right\rangle \\
&+t f(y)+(1-t) f\left(x_{\epsilon}\right)-f\left(x_{\epsilon}\right) \\
& \geq\left\langle T x_{\epsilon}, \eta\left(t y+(1-t) x_{\epsilon}, x_{\epsilon}\right)\right\rangle+f\left(t y+(1-t) x_{\epsilon}\right) \\
&-f\left(x_{\epsilon}\right) \\
& \geq 0,
\end{aligned}
$$

for all $y \in K$. This implies that

$$
\left\langle T x_{\epsilon}, \eta\left(y, x_{\epsilon}\right)\right\rangle+f(y)-f\left(x_{\epsilon}\right) \geq 0,
$$

for all $y \in K$. Therefore, $x_{\epsilon} \in K$ is a solution of the problem (9). This completes the proof.

It is easy to see that the relaxed $\eta$ - $\alpha$ monotonicity implies the weakly relaxed $\eta-\alpha$ monotonicity. So, Theorem 15 can be deduced to the following corollary.

Corollary 16. Let $K$ be a nonempty unbounded closed convex subset of a real reflexive Banach space $E$, and let $E^{*}$ be the dual space of $E$. Suppose that $T: K \rightarrow E^{*}$ is an $\eta$-hemicontinuous and relaxed $\eta-\alpha$ monotone mapping and $f: K \rightarrow \mathbb{R} \cup\{\infty\}$ is a proper convex lower semicontinuous function. If the following conditions hold:

(a) $\eta(x, y)+\eta(y, x)=0$ for all $x, y \in K$,

(b) for any fixed $y, z \in K$, the mapping $x \mapsto\langle T z, \eta(x, y)\rangle$ is convex and lower semicontinuous function,

(c) $\alpha$ is weakly lower semicontinuous,

(d) $T$ is weakly $\eta$-coercive with respect to $f$,

then the problem (9) is solvable.

Since the $\eta$-coercivity with respect to $f$ implies that the weakly $\eta$-coercivity with respect to $f$, we can utilize Corollary 16 to the result of Fang and Huang [16].
Corollary 17 (Theorem $2.3[16]$ ). Let $K$ be a nonempty unbounded closed convex subset of a real reflexive Banach space $E$, and let $E^{*}$ be the dual space of $E$. Suppose that $T: K \rightarrow E^{*}$ is an $\eta$-hemicontinuous and relaxed $\eta$ - $\alpha$ monotone mapping and $f: K \rightarrow \mathbb{R} \cup\{\infty\}$ is a proper convex lower semicontinuous function. If the following conditions hold:

(a) $\eta(x, y)+\eta(y, x)=0$ for all $x, y \in K$,

(b) for any fixed $y, z \in K$, the mapping $x \mapsto\langle T z, \eta(x, y)\rangle$ is convex and lower semicontinuous function,

(c) $\alpha$ is weakly lower semicontinuous,

(d) $T$ is $\eta$-coercive with respect to $f$,

then the problem (9) is solvable.

Remark 18. Theorems 11, 12, and 15 are improvement of the results of Fang and Huang [16] from the corresponding results of variational-like inequality problems for relaxed $\eta-\alpha$ monotone mapping to weakly relaxed $\eta-\alpha$ monotone mapping. Also, these results are extension of the known results of Hartman and Stampacchia [22] and corresponding results in $[14,19,23]$.

\section{Variational-Like Inequalities Problems with Weakly Relaxed $\eta$ - $\alpha$ Semimonotone Mapping}

Through this section, let $E$ be an arbitrary Banach space with its dual space $E^{*}$, let $E^{* *}$ denote the dual space of $E^{*}$, and let $K$ be a nonempty closed convex subset of $E^{* *}$.

Definition 19. A mapping $A: K \times K \rightarrow E^{* *}$ is said to be weakly relaxed $\eta-\alpha$ semimonotone if the following conditions hold:

(a) for each fixed $u \in K, A(u, \cdot)$ is weakly relaxed $\eta-\alpha$ monotone; that is, there exist mappings $\eta: K \times K \rightarrow$ $E^{*}$ and $\alpha: E^{* *} \rightarrow \mathbb{R}$ such that

$$
\begin{gathered}
\lim _{t \rightarrow 0^{+}} \alpha(t x)=0, \\
\lim _{t \rightarrow 0^{+}} \frac{d}{d t} \alpha(t x)=0,
\end{gathered}
$$

for all $t>0$ and $x \in E^{* *}$ and

$$
\begin{array}{r}
\langle A(u, v)-A(u, w), \eta(v, w)\rangle \geq \alpha(v-w) \\
\forall v, w \in K ;
\end{array}
$$

(b) for each fixed $v \in K, A(\cdot, v)$ is completely continuous; that is, for any net $\left\{u_{\beta}\right\}$ with $u_{\beta}$ converges to $u_{0}$ in $\sigma\left(E^{* *}, E^{*}\right)$, we have that $\left\{A\left(u_{\beta}, v\right)\right\}$ converges to $A\left(u_{0}, v\right)$ in the norm topology of $E^{*}$.

Remark 20. We obtain that relaxed $\eta-\alpha$ semimonotonicity due to Fang and Huang [16] implies weakly relaxed $\eta-\alpha$ semimonotonicity. Therefore, the class of relaxed $\eta$ - $\alpha$ semimonotone mappings is subclass of the class of weakly relaxed $\eta-\alpha$ semimonotone mappings. 
Let $A: K \times K \rightarrow E^{* *}$ and $\eta: K \times K \rightarrow E^{*}$ be two mappings and $f: K \rightarrow \mathbb{R} \cup\{\infty\}$ is a proper convex lower-semicontinuous function. We consider the following problem:

$$
\text { find } u \in K
$$

$$
\begin{array}{r}
\text { such that }\langle A(u, u), \eta(v, u)\rangle+f(v)-f(u) \geq 0, \\
\forall v \in K .
\end{array}
$$

Theorem 21. Let $E$ be a real Banach space and let $K \subset E^{* *}$ be a nonempty bounded closed convex set. Suppose that $A: K \times$ $K \rightarrow E^{* *}$ is a weakly relaxed $\eta-\alpha$ semimonotone mapping and let $f: K \rightarrow \mathbb{R} \cup\{\infty\}$ be a proper convex lower semicontinuous function. If the following conditions hold:

(a) $\eta(x, y)+\eta(y, x)=0$ for all $x, y \in K$,

(b) for any fixed $y, z, w \in K$, the mapping $x \mapsto\langle A(z$, $w), \eta(x, y)\rangle$ is convex and lower semicontinuous function,

(c) for each $x \in K, A(x, \cdot): K \rightarrow E^{* *}$ is finite-dimensional continuous; that is, for any finite-dimensional subspace $F \subset E^{* *}, A(x, \cdot): K \cap F \rightarrow E^{* *}$ is continuous,

(d) $\alpha$ is convex lower semicontinuous,

then the problem (43) is solvable.

Proof. Let $F \subset E^{* *}$ be a finite-dimensional subspace with $K_{F}:=F \cap K \neq \emptyset$. For each $w \in K$, consider the following problem:

$$
\text { find } u_{0} \in K_{F}
$$

$$
\begin{array}{r}
\text { such that }\left\langle A\left(w, u_{0}\right), \eta\left(v, u_{0}\right)\right\rangle+f(v)-f\left(u_{0}\right) \geq 0 \\
\forall v \in K_{F} .
\end{array}
$$

It follows from $K_{F}$ being bounded closed and convex and $A(w, \cdot)$ being continuous on $K_{F}$ and weakly relaxed $\eta-\alpha$ monotone for each fixed $w \in K$ that from Theorem 12, we obtain that problem (44) has a solution $u_{0} \in K_{F}$.

Next, define a set-valued mapping $T: K_{F} \rightarrow 2^{K_{F}}$ as follows:

$$
\begin{gathered}
T w=\left\{u \in K_{F}:\langle A(w, u), \eta(v, u)\rangle+f(v)-f(u)\right. \\
\left.\geq 0, \forall v \in K_{F}\right\}, \quad \forall w \in K_{F} .
\end{gathered}
$$

By Theorem 11, we get that, for each $w \in K_{F}$,

$$
\begin{gathered}
T w=\left\{u \in K_{F}:\langle A(w, u), \eta(v, u)\rangle+f(v)-f(u)\right. \\
\left.\geq \alpha(v-u), \forall v \in K_{F}\right\} .
\end{gathered}
$$

It is known that every convex lower-semicontinuous function in Banach spaces is weakly lower semicontinuous. Therefore, condition (b) and the proper convex lower semicontinuity of $f$ and $\alpha$ implies that $T$ has nonempty bounded closed and convex values. By the complete continuity of $A(\cdot, u)$, we have that $T$ is upper semicontinuous.
Using the Kakutani-Fan-Glicksberg fixed-point theorem, we obtain that $T$ has a fixed-point $w_{0} \in K_{F}$ and thus

$$
\left\langle A\left(w_{0}, w_{0}\right), \eta\left(v, w_{0}\right)\right\rangle+f(v)-f\left(w_{0}\right) \geq 0, \quad \forall v \in K_{F} .
$$

Now, define

$$
\begin{gathered}
\mathscr{F}:=\left\{F \subset E^{* *}: F \text { is finite dimensional with } F \cap K \neq \emptyset\right\}, \\
W_{F}:=\{u \in K:\langle A(u, v), \eta(v, u)\rangle+f(v)-f(u) \\
\left.\geq \alpha(v-u), \forall v \in K_{F}\right\}, \quad \forall F \in \mathscr{F} .
\end{gathered}
$$

From (47), using Theorem 11, we obtain that $W_{F}$ is nonempty and bounded. Here, we denote $\overline{W_{F}}$ by the $\sigma\left(E^{* *}, E^{*}\right)$-closure of $W_{F}$ in $E^{* *}$ and thus $\overline{W_{F}}$ is $\sigma\left(E^{* *}, E^{*}\right)$-compact in $E^{* *}$.

It is known that, for any $F_{i} \in \mathscr{F}, i \in \mathbb{N}$, we have $W_{\cap_{i} F_{i}} \subset$ $\cap W_{F_{i}}$. Therefore, $\left\{\overline{W_{F}}: F \in \mathscr{F}\right\}$ has the finite intersection property; that is,

$$
\bigcap_{F \in \mathscr{F}} \overline{W_{F}} \neq \emptyset .
$$

Next, we show that, for $u \in \bigcap_{F \in \mathscr{F}} \overline{W_{F}}$,

$$
\langle A(u, u), \eta(v, u)\rangle+f(v)-f(u) \geq 0, \quad \forall v \in K .
$$

Indeed, for $v \in K$, let $F \in \mathscr{F}$ be such that $v \in K_{F}$ and $u \in K_{F}$. Then, there exists a net $\left\{u_{\beta}\right\}$ in $W_{F}$ such that $u_{\beta}$ converges to $u$ in $\sigma\left(E^{* *}, E^{*}\right)$. From the definition of $W_{F}$, we have

$$
\left\langle A\left(u_{\beta}, v\right), \eta\left(v, u_{\beta}\right)\right\rangle+f(v)-f\left(u_{\beta}\right) \geq \alpha\left(v-u_{\beta}\right) .
$$

By the complete continuity of $A(\cdot, v)$ and the proper convex lower semicontinuity of $f$ and $\alpha$, we get

$$
\langle A(u, v), \eta(v, u)\rangle+f(v)-f(u) \geq \alpha(v-u), \quad \forall v \in K .
$$

Again, using Theorem 11, we conclude that

$$
\langle A(u, v), \eta(v, u)\rangle+f(v)-f(u) \geq 0, \quad \forall v \in K .
$$

This implies that $u$ is a solution of the problem (43). This completes the proof.

Theorem 22. Let $E$ be a real Banach space and let $K \subset E^{* *}$ be a nonempty unbounded closed convex set. Suppose that $A: K \times$ $K \rightarrow E^{* *}$ is a weakly relaxed $\eta-\alpha$ semimonotone mapping and let $f: K \rightarrow \mathbb{R} \cup\{\infty\}$ be a proper convex lower semicontinuous function. If the following conditions hold:

(a) $\eta(x, y)+\eta(y, x)=0$ for all $x, y \in K$,

(b) for any fixed $y, z, w \in K$, the mapping $x \mapsto\langle A(z$, $w), \eta(x, y)\rangle$ is convex and lower semicontinuous function, 
(c) for each $x \in K, A(x, \cdot): K \rightarrow E^{*}$ is finite-dimensional continuous,

(d) $\alpha$ is convex lower semicontinuous,

(e) there exists a point $u_{0} \in K$ such that $\lim _{\inf }\|u\| \rightarrow \infty$ $\left[\left\langle A(u, u), \eta\left(u, u_{0}\right)\right\rangle+f(u)-f\left(u_{0}\right)\right]>0$,

then the problem (43) is solvable.

Proof. For $\epsilon>0$, we denote by $B_{\epsilon}$ the closed ball with radius $\epsilon$ and center at 0 in $E^{* *}$. By Theorem 21, the problem

$$
\langle A(u, u), \eta(v, u)\rangle+f(v)-f(u) \geq 0, \quad \forall v \in B_{\epsilon}
$$

has a solution $u_{\epsilon} \in B_{\epsilon}$.

Let $\epsilon$ be large enough such that $u_{0} \in B_{\epsilon}$. Therefore,

$$
\left\langle A\left(u_{\epsilon}, u_{\epsilon}\right), \eta\left(u_{0}, u_{\epsilon}\right)\right\rangle+f\left(u_{0}\right)-f\left(u_{\epsilon}\right) \geq 0 .
$$

By condition (e), we get that $\left\{u_{\epsilon}\right\}$ is bounded. So, we may suppose that $u_{\epsilon}$ converges to $u$ in $\sigma\left(E^{* *}, E^{*}\right)$ as $\epsilon \rightarrow \infty$. It follows from Theorem 11 that

$$
\begin{array}{r}
\left\langle A\left(u_{\epsilon}, v\right), \eta\left(v, u_{\epsilon}\right)\right\rangle+f(v)-f\left(u_{\epsilon}\right) \geq \alpha\left(v-u_{\epsilon}\right), \\
\forall v \in K .
\end{array}
$$

Letting $\epsilon \rightarrow \infty$, we have

$$
\langle A(u, v), \eta(v, u)\rangle+f(v)-f(u) \geq \alpha(v-u), \quad \forall v \in K .
$$

Again from Theorem 11, we get

$$
\langle A(u, u), \eta(v, u)\rangle+f(v)-f(u) \geq 0, \quad \forall v \in K .
$$

This show that $u \in K$ is a solution of the problem (43). This completes the proof.

Remark 23. Theorems 21 and 22 extend and improve Theorems 3.1 and 3.2 of Fang and Huang [16] and Theorems 2.1 to 2.6 of Chen [7].

\section{Acknowledgments}

Marwan Amin Kutbi gratefully acknowledges the support from the Deanship of Scientific Research (DSR) at King Abdulaziz University (KAU) during this research. Wutiphol Sintunavarat gratefully acknowledges Thammasat University for the support of this research.

\section{References}

[1] M.-R. Bai, S.-Z. Zhou, and G.-Y. Ni, "On the generalized monotonicity of variational inequalities," Computers \& Mathematics with Applications, vol. 53, no. 6, pp. 910-917, 2007.

[2] S.-S. Chang, B. S. Lee, and Y.-Q. Chen, "Variational inequalities for monotone operators in nonreflexive Banach spaces," Applied Mathematics Letters, vol. 8, no. 6, pp. 29-34, 1995.
[3] L. B. dos Santos, G. Ruiz-Garzón, M. A. Rojas-Medar, and A. Rufián-Lizana, "Some relations between variational-like inequality problems and vectorial optimization problems in Banach spaces," Computers \& Mathematics with Applications, vol. 55, no. 8, pp. 1808-1814, 2008.

[4] Y.-b. Xiao and N.-j. Huang, "Generalized quasi-variationallike hemivariational inequalities," Nonlinear Analysis: Theory, Methods \& Applications, vol. 69, no. 2, pp. 637-646, 2008.

[5] Y. Zhao and Z. Xia, "On the existence of solutions to generalized vector variational-like inequalities," Nonlinear Analysis: Theory, Methods \& Applications, vol. 64, no. 9, pp. 2075-2083, 2006.

[6] Y. Zhao and Z. Xia, "Existence results for systems of vector variational-like inequalities," Nonlinear Analysis: Real World Applications, vol. 8, no. 5, pp. 1370-1378, 2007.

[7] Y.-Q. Chen, "On the semi-monotone operator theory and applications," Journal of Mathematical Analysis and Applications, vol. 231, no. 1, pp. 177-192, 1999.

[8] L. C. Ceng and J. C. Yao, “On generalized variational-like inequalities with generalized monotone multivalued mappings," Applied Mathematics Letters, vol. 22, no. 3, pp. 428-434, 2009.

[9] R. W. Cottle and J. C. Yao, "Pseudomonotone complementarity problems in Hilbert spaces," Journal of Optimization Theory and Applications, vol. 78, no. 2, pp. 281-295, 1992.

[10] X. P. Ding, "Existence and algorithm of solutions for generalized mixed implicit quasi-variational inequalities," Applied Mathematics and Computation, vol. 113, no. 1, pp. 67-80, 2000.

[11] R. Glowinski, J.-L. Lions, and R. Trémolières, Numerical Analysis of Variational Inequalities, North-Holland, Amsterdam, Holland, 1981.

[12] D. Goeleven and D. Motreanu, "Eigenvalue and dynamic problems for variational and hemivariational inequalities," Communications on Applied Nonlinear Analysis, vol. 3, no. 4, pp. 1-21, 1996.

[13] R. U. Verma, "On generalized variational inequalities involving relaxed Lipschitz and relaxed monotone operators," Journal of Mathematical Analysis and Applications, vol. 213, no. 1, pp. 387392, 1997.

[14] R. U. Verma, "On monotone nonlinear variational inequality problems," Commentationes Mathematicae Universitatis Carolinae, vol. 39, no. 1, pp. 91-98, 1998.

[15] X. Q. Yang and G. Y. Chen, "A class of nonconvex functions and pre-variational inequalities," Journal of Mathematical Analysis and Applications, vol. 169, no. 2, pp. 359-373, 1992.

[16] Y. P. Fang and N. J. Huang, "Variational-like inequalities with generalized monotone mappings in Banach spaces," Journal of Optimization Theory and Applications, vol. 118, no. 2, pp. 327338, 2003.

[17] W. Sintunavarat, "Mixed equilibrium problems with weakly relaxed $\alpha$-monotone bi-function in Banach spaces," Journal of Function Spaces and Applications, vol. 2013, Article ID 374268, 5 pages, 2013.

[18] J. C. Yao, "Existence of generalized variational inequalities," Operations Research Letters, vol. 15, no. 1, pp. 35-40, 1994.

[19] D. Goeleven and D. Motreanu, "Eigenvalue and dynamic problems for variational and hemivariational inequalities," Communications on Applied Nonlinear Analysis, vol. 3, no. 4, pp. 1-21, 1996.

[20] R. U. Verma, "Nonlinear variational inequalities on convex subsets of Banach spaces," Applied Mathematics Letters, vol. 10, no. 4, pp. 25-27, 1997. 
[21] K. Fan, "A generalization of Tychonoff's fixed point theorem," Mathematische Annalen, vol. 142, pp. 305-310, 1960-1961.

[22] G. J. Hartman and G. Stampacchia, "On some nonlinear elliptic differential functional equations," Acta Mathematica, vol. 115, pp. 271-310, 1996.

[23] A. H. Siddiqi, Q. H. Ansari, and K. R. Kazmi, "On nonlinear variational inequalities," Indian Journal of Pure and Applied Mathematics, vol. 25, no. 9, pp. 969-973, 1994. 


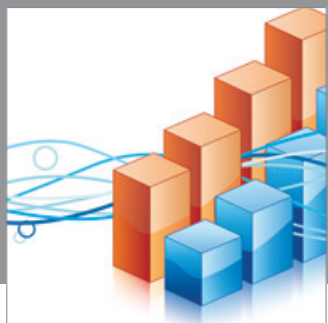

Advances in

Operations Research

mansans

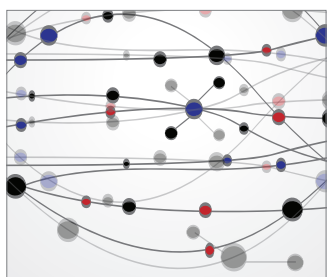

The Scientific World Journal
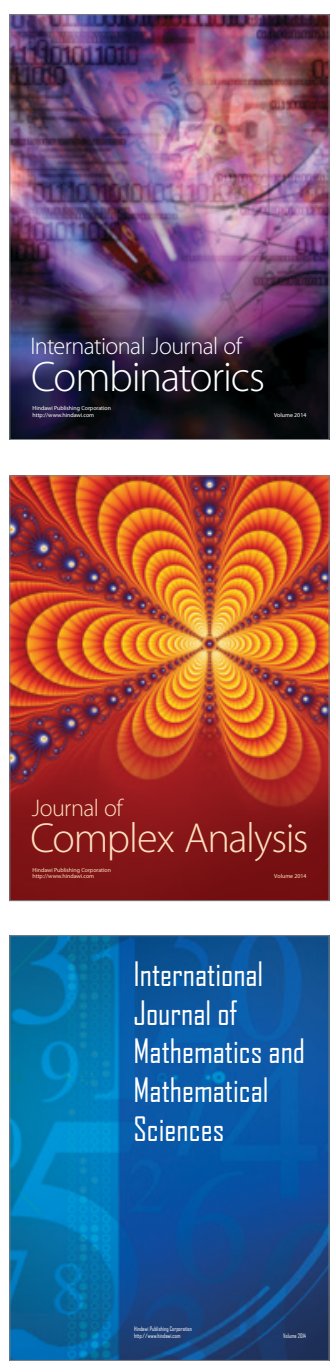
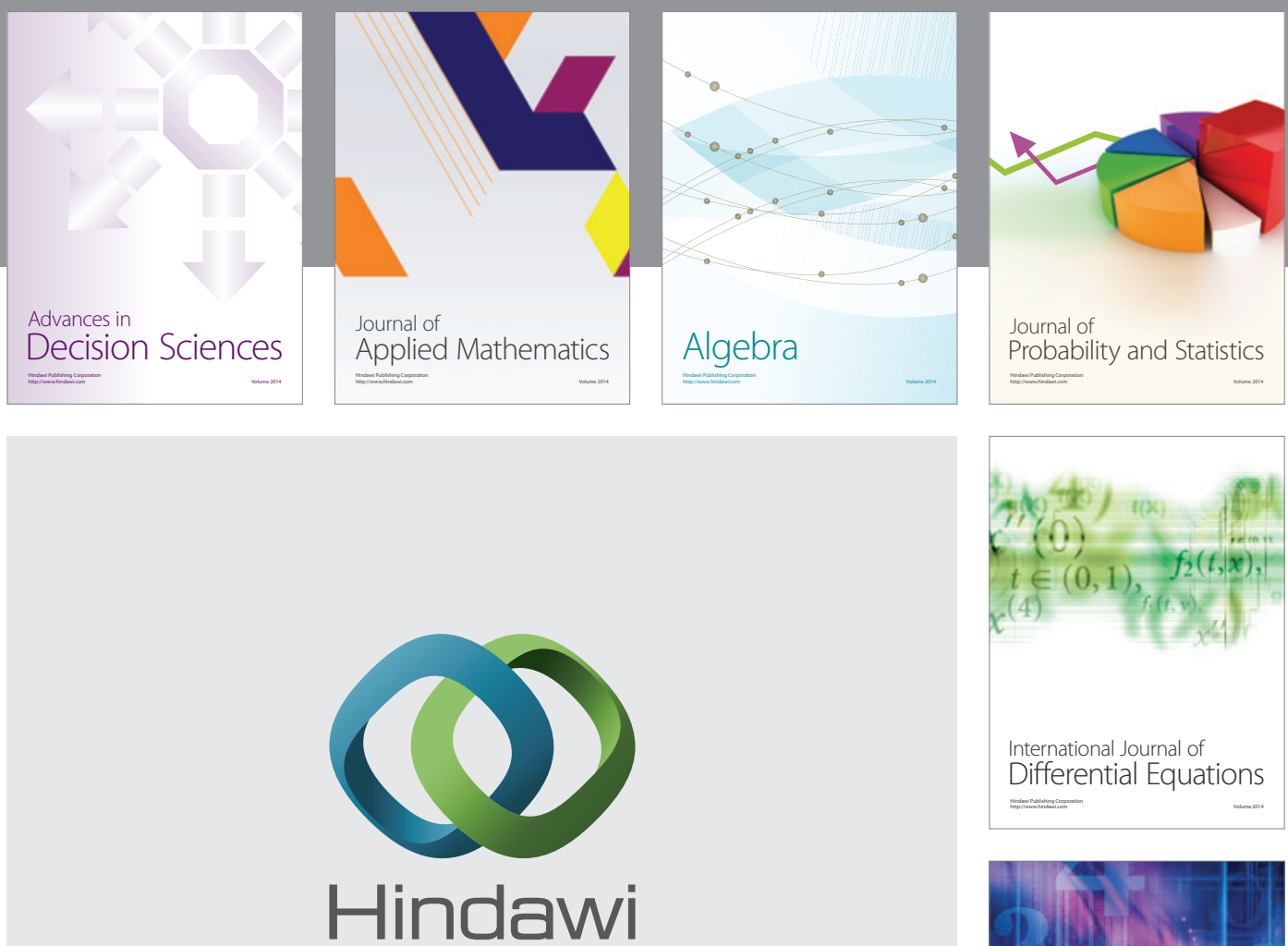

Submit your manuscripts at http://www.hindawi.com


Journal of

Function Spaces

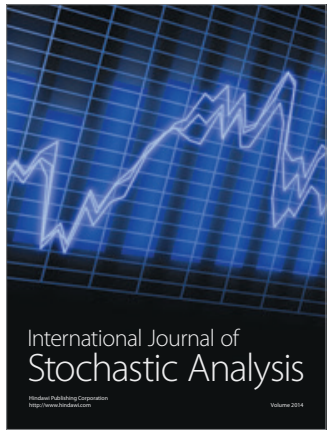

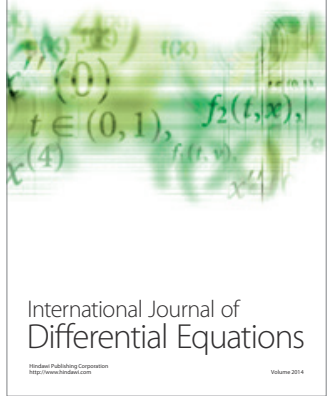
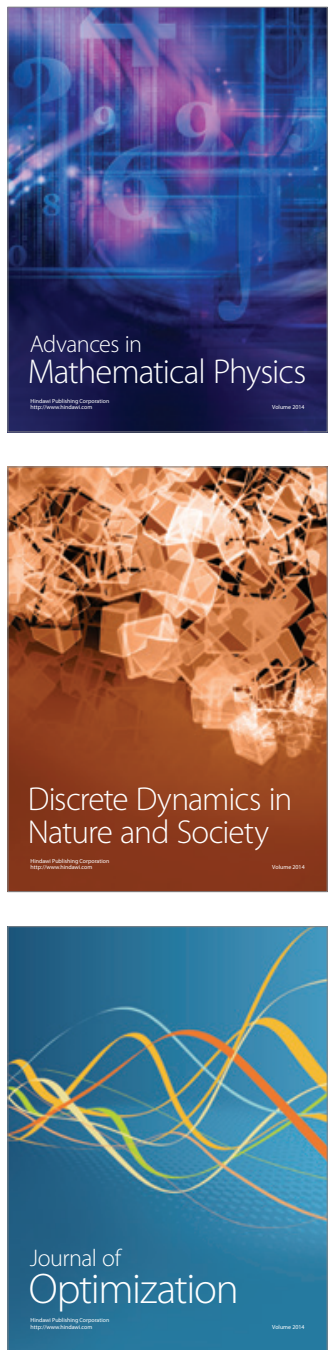